\title{
The Elser nuclei sum revisited
}

\author{
Darij Grinberg \\ Drexel University, Philadelphia, PA, USA
}

received $22^{\text {nd }}$ Dec. 2020, revised 12 $2^{\text {th }}$ Jan. 2021, $28^{\text {th }}$ Apr. 2021, accepted $4^{\text {th }}$ May 2021.

Fix a finite undirected graph $\Gamma$ and a vertex $v$ of $\Gamma$. Let $E$ be the set of edges of $\Gamma$. We call a subset $F$ of $E$ pandemic if each edge of $\Gamma$ has at least one endpoint that can be connected to $v$ by an $F$-path (i.e., a path using edges from $F$ only). In 1984, Elser showed that the sum of $(-1)^{|F|}$ over all pandemic subsets $F$ of $E$ is 0 if $E \neq \varnothing$. We give a simple proof of this result via a sign-reversing involution, and discuss variants, generalizations and a refinement using discrete Morse theory.

Keywords: graph, simplicial complex, alternating sum, discrete Morse theory

In Elser 1984, Veit Elser studied the probabilities of clusters forming when $n$ points are sampled randomly in a $d$-dimensional volume. In the process, he found a purely graph-theoretical lemma Elser, 1984, Lemma 1), which served a crucial role in his work. For decades, the lemma stayed hidden from the eyes of combinatorialists in a physics journal, until it resurfaced in recent work Dorpalen-Barry et al. 2021) by Dorpalen-Barry, Hettle, Livingston, Martin, Nasr, Vega and Whitlatch. In this note, I will show a simpler proof of the lemma using a sign-reversing involution. The proof also suggests multiple venues of generalization that I will explore in the later sections; one extends the lemma to a statement about arbitrary antimatroids (and even a wider setting). Finally, I will strengthen the lemma to a Morse-theoretical result, stating the collapsibility of a certain simplicial complex. Some open questions will be posed.

\section{Remark on alternative versions}

The current version of this paper is written with a combinatorially experienced reader in mind. The previous arXiv version Grinberg (2021) includes more details in the proofs.

\section{Elser's result}

Let us first introduce our setting, which is slightly more general (and perhaps also simpler) than that used in Elser (1984). (In Section 4 , we will move to a more general setup.)

We fix an arbitrary graph $\Gamma$ with vertex set $V$ and edge set $E$. Here, "graph" means "finite undirected multigraph" - i.e., it can have self-loops and parallel edges, but it has finitely many vertices and edges, and its edges are undirected.

We fix a vertex $v \in V$.

If $F \subseteq E$, then an $F$-path shall mean a path of $\Gamma$ such that all edges of the path belong to $F$.

ISSN 1365-8050 @ 2021 by the author(s) Distributed under a Creative Commons Attribution 4.0 International License 
If $e \in E$ is any edge and $F \subseteq E$ is any subset, then we say that $F$ infects $e$ if there exists an $F$-path from $v$ to some endpoint of $e$. (The terminology is inspired by the idea of an infectious disease starting in the vertex $v$ and being transmitted along edges.) (i)

A subset $F \subseteq E$ is said to be pandemic if it infects each edge $e \in E$.

Example 1.1. Let $\Gamma$ be the following graph:

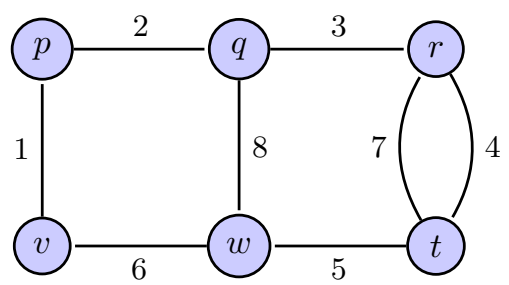

(where the vertex $v$ is the vertex labelled $v$ ). Then, for example, the set $\{1,2\} \subseteq E$ infects edges $1,2,3,6,8$ (but none of the other edges). The set $\{1,2,5\}$ infects the same edges as $\{1,2\}$ (indeed, the additional edge 5 does not increase its infectiousness, since it is not on any $\{1,2,5\}$-path from $v$ ). The set $\{1,2,3\}$ infects every edge other than 5 . The set $\{1,2,3,4\}$ infects each edge, and thus is pandemic.

Now, we can state our version of (Elser, 1984, Lemma 1):

Theorem 1.2. Assume that $E \neq \varnothing$. Then,

$$
\sum_{\substack{F \subseteq E \text { is } \\ \text { pandemic }}}(-1)^{|F|}=0 .
$$

Example 1.3. Let $\Gamma$ be the following graph:

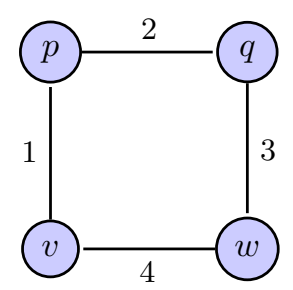

(where the vertex $v$ is the vertex labelled $v$ ). Then, the pandemic subsets of $E$ are the sets

$$
\{1,2\},\{1,4\},\{3,4\},\{1,2,3\},\{1,3,4\},\{1,2,4\},\{2,3,4\},\{1,2,3,4\} .
$$

The sizes of these subsets are $2,2,2,3,3,3,3,4$, respectively. Hence, (1) says that

$$
(-1)^{2}+(-1)^{2}+(-1)^{2}+(-1)^{3}+(-1)^{3}+(-1)^{3}+(-1)^{3}+(-1)^{4}=0 .
$$

(i) Note that if an edge $e$ contains the vertex $v$, then any subset $F$ of $E$ (even the empty one) infects $e$, since there is a trivial (edgeless) $F$-path from $v$ to $v$. 
We note that the equality (11) can be restated as "there are equally many pandemic subsets $F \subseteq E$ of even size and pandemic subsets $F \subseteq E$ of odd size". Thus, in particular, the number of all pandemic subsets $F$ of $E$ is even (when $E \neq \varnothing$ ).

Remark 1.4. Theorem 1.2 is a bit more general than (Elsen, 1984, Lemma 1). To see why, we assume that the graph $\Gamma$ is connected and simple (i.e., has no self-loops and parallel edges). Then, a nucleus is defined in Elsen (1984) as a subgraph $N$ of $\Gamma$ with the properties that

1. the subgraph $N$ is connected, and

2. each edge of $\Gamma$ has at least one endpoint in $N$.

Given a subgraph $N$ of $\Gamma$, we let $\mathrm{E}(N)$ denote the set of all edges of $N$. Now, Elser, 1984, Lemma 1) claims that if $E \neq \varnothing$, then

$$
\sum_{\begin{array}{c}
N \text { is a nucleus } \\
\text { containing } v
\end{array}}(-1)^{|\mathrm{E}(N)|}=0 .
$$

But this is equivalent to (7), because there is a bijection

$$
\begin{aligned}
\{\text { nuclei containing } v\} & \rightarrow\{\text { pandemic subsets } F \subseteq E\}, \\
N & \mapsto \mathrm{E}(N) .
\end{aligned}
$$

We leave it to the reader to check this in detail; what needs to be checked are the following three statements:

- If $N$ is a nucleus containing $v$, then $\mathrm{E}(N)$ is a pandemic subset of $E$.

- Every nucleus $N$ containing $v$ is uniquely determined by the set $\mathrm{E}(N)$. (Indeed, since a nucleus has to be connected, each of its vertices must be an endpoint of one of its edges, unless its only vertex is $v$.)

- If $F$ is a pandemic subset of $E$, then there is a nucleus $N$ containing $v$ such that $\mathrm{E}(N)=F$. (Indeed, $N$ can be defined as the subgraph of $\Gamma$ whose vertices are the endpoints of all edges in $F$ as well as the vertex $v$, and whose edges are the edges in $F$. To see that this subgraph $N$ is connected, it suffices to argue that each of its vertices has a path to v; but this follows from the definition of "pandemic", since each vertex of $N$ other than $v$ belongs to at least one edge in $F$.)

Thus, Theorem 1.2 is equivalent to (Elser, 1984, Lemma 1) in the case when $\Gamma$ is connected and simple.

Remark 1.5. It might appear more natural to talk about a subset $F \subseteq E$ infecting a vertex rather than an edge. (Namely, we can say that $F$ infects a vertex $w$ if there is an F-path from $v$ to $w$.) However, the analogue of Theorem 1.2 in which pandemicity is defined via infecting all vertices is not true. The graph of Example 1.3 provides a counterexample. 


\section{The proof}

\subsection{Set-theoretical notions}

We shall first introduce some concepts and notations pertaining to arbitrary sets. They will aid us in proving Theorem 1.2, and also in generalizing it later on.

Definition 2.1. Let $A$ and $B$ be two sets. Then, we say that $A \prec B$ if we have $B=A \cup\{b\}$ for some $b \in B \backslash A$. Equivalently, $A \prec B$ holds if and only if $A \subseteq B$ and $|B \backslash A|=1$.

Definition 2.2. We let $\mathcal{P}(E)$ denote the power set of $E$ (that is, the set of all subsets of $E$ ).

Definition 2.3. Let $\mathcal{A}$ be a set. A map $\mu: \mathcal{A} \rightarrow \mathcal{A}$ is said to be an involution if $\mu \circ \mu=\operatorname{id}_{\mathcal{A}}$.

Definition 2.4. Let $\mathcal{A}$ be a subset of $\mathcal{P}(E)$. A complete matching of $\mathcal{A}$ shall mean an involution $\mu: \mathcal{A} \rightarrow$ $\mathcal{A}$ with the property that each $F \in \mathcal{A}$ satisfies

$$
\text { either } \mu(F) \prec F \text { or } F \prec \mu(F) \text {. }
$$

The following simple fact abstracts an idea that will be used at least twice:

Lemma 2.5. Let $\mathcal{A}$ be a subset of $\mathcal{P}(E)$. Let $\mu: \mathcal{A} \rightarrow \mathcal{A}$ be a complete matching of $\mathcal{A}$. Then,

$$
\sum_{F \in \mathcal{A}}(-1)^{|F|}=0
$$

Proof: This is a standard argument in enumerative combinatorics (see Sagan, 2020, (2.3)) or Benjamin and Quinn (2008) for a more general viewpoint). Here is the proof: For each $F \in \mathcal{A}$, we have $|\mu(F)|=$ $|F| \pm 1$ (because (2) shows that the sets $F$ and $\mu(F)$ differ in exactly one element) and thus $(-1)^{|\mu(F)|}+$ $(-1)^{|F|}=0$. This shows, in particular, that $\mu$ has no fixed points. Thus, the involution $\mu$ partitions the set $\mathcal{A}$ into 2-element subsets $\{F, \mu(F)\}$. Each such 2-element subset contributes $(-1)^{|\mu(F)|}+(-1)^{|F|}=0$ to the sum on the left hand side of (3). Hence, this sum is 0 . This proves Lemma 2.5.

\subsection{Shades}

Next, we shall introduce the notion of a shade; this will be crucial to proving and generalizing Theorem 1.2 .

Definition 2.6. Let $F$ be a subset of $E$. Then, we define a subset Shade $F$ of $E$ by

$$
\text { Shade } F=\{e \in E \mid F \text { infects } e\} .
$$

We refer to Shade $F$ as the shade of $F$.

Thus, the shade of a subset $F \subseteq E$ is the set of all edges of $\Gamma$ that are infected by $F$. (In more standard graph-theoretical lingo, this means that Shade $F$ is the set of edges that contain at least one vertex of the connected component containing $v$ of the graph $(V, F)$.)

Example 2.7. In Example 1.1, we have Shade $\{1,2\}=\{1,2,3,6,8\}$ and Shade $\{1\}=\{1,2,6\}$ and Shade $\{8\}=\{1,6\}$.

The following property of shades is rather obvious: 
Lemma 2.8. Let $A$ and $B$ be two subsets of $E$ such that $A \subseteq B$. Then, Shade $A \subseteq$ Shade $B$.

The major property of shades that we will need is the following:

Lemma 2.9. Let $F$ be a subset of E. Let $u \in E$ be such that $u \notin$ Shade $F$. Then,

$$
\text { Shade }(F \cup\{u\})=\text { Shade } F
$$

and

$$
\text { Shade }(F \backslash\{u\})=\text { Shade } F \text {. }
$$

Proof: There is no $F$-path from $v$ to any endpoint of $u$ (since $u \notin$ Shade $F$ ). Hence, any $(F \cup\{u\}$ )-path that starts at $v$ must be an $F$-path (as it would otherwise use the edge $u$ and thus contain an $F$-path from $v$ to some endpoint of $u)$. This entails Shade $(F \cup\{u\}) \subseteq$ Shade $F$. Combined with the opposite inclusion (which follows from Lemma 2.8), this yields (5).

Also, Lemma 2.8 yields Shade $(F \backslash\{u\}) \subseteq$ Shade $F$, so that $u \notin$ Shade $(F \backslash\{u\})$. Hence, (6) follows by applying (5) to $F \backslash\{u\}$ instead of $F$.

\subsection{A slightly more general claim}

Lemma 2.9 might not look very powerful, but it contains all we need to prove Theorem 1.2 . Better yet, we shall prove the following slightly more general version of Theorem 1.2 .

Theorem 2.10. Let $G$ be any subset of $E$. Assume that $E \neq \varnothing$. Then,

$$
\sum_{\substack{F \subseteq E ; \\ G \subseteq \text { Shade } F}}(-1)^{|F|}=0 .
$$

We will soon prove Theorem 2.10 and explain how Theorem 1.2 follows from it. First, however, let us give an equivalent (but slightly easier to prove) version of Theorem 2.10:

Theorem 2.11. Let $G$ be any subset of $E$. Then,

$$
\sum_{\substack{F \subseteq E ; \\ G \nsubseteq \text { Shade } F}}(-1)^{|F|}=0 .
$$

Proof: Let

$$
\mathcal{A}=\{F \subseteq E \mid G \nsubseteq \text { Shade } F\}
$$

Thus, $\mathcal{A}$ is a subset of $\mathcal{P}(E)$, and each $F \in \mathcal{A}$ satisfies $G \nsubseteq$ Shade $F$.

We equip the finite set $E$ with a total order (chosen arbitrarily, but fixed henceforth). If $F \in \mathcal{A}$, then we define $\varepsilon(F)$ to be the smallest edge $e \in G \backslash$ Shade $F$. (Such an edge exists, since $F \in \mathcal{A}$ entails $G \nsubseteq$ Shade $F$ and thus $G \backslash$ Shade $F \neq \varnothing$.)

For any $F \in \mathcal{A}$, we have $\varepsilon(F) \notin$ Shade $F$ (by the definition of $\varepsilon(F)$ ). Thus, any $F \in \mathcal{A}$ satisfies Shade $(F \cup\{\varepsilon(F)\})=$ Shade $F$ (by (5)) and Shade $(F \backslash\{\varepsilon(F)\})=$ Shade $F$ (by (6)). In other words, if we replace a set $F \in \mathcal{A}$ by $F \cup\{\varepsilon(F)\}$ or $F \backslash\{\varepsilon(F)\}$, then Shade $F$ does not change. Hence, $\varepsilon(F)$ does not change either (since $\varepsilon(F)$ depends only on Shade $F$, but not on $F$ itself). Furthermore, the 
resulting set $(F \cup\{\varepsilon(F)\}$ or $F \backslash\{\varepsilon(F)\})$ still belongs to $\mathcal{A}$ (since Shade $F$ has not changed). Thus, we can define a map

$$
\begin{aligned}
\mu: \mathcal{A} & \rightarrow \mathcal{A}, \\
F & \mapsto \begin{cases}F \cup\{\varepsilon(F)\}, & \text { if } \varepsilon(F) \notin F ; \\
F \backslash\{\varepsilon(F)\}, & \text { if } \varepsilon(F) \in F .\end{cases}
\end{aligned}
$$

Clearly, this map $\mu$ is an involution (since we have shown that $\varepsilon(F)$ does not change when we replace $F$ by $\mu(F)$ ). Moreover, this map $\mu$ is a complete matching (since each $F \in \mathcal{A}$ satisfies $F \prec \mu(F)$ if $\varepsilon(F) \notin F$, and satisfies $\mu(F) \prec F$ otherwise). Hence, Lemma 2.5 yields $\sum_{F \in \mathcal{A}}(-1)^{|F|}=0$. In view of how we defined $\mathcal{A}$, this is equivalent to (7). Thus, (7) is proven.

In order to derive Theorem 2.10 from Theorem 2.11, we need the following innocent lemma:

Lemma 2.12. Let $U$ be a finite set with $U \neq \varnothing$. Then,

$$
\sum_{F \subseteq U}(-1)^{|F|}=0
$$

Proof: This is an easy (and well-known) consequence of Lemma 2.5. It also follows from the well-known binomial identity $\sum_{k=0}^{n}(-1)^{k}\left(\begin{array}{l}n \\ k\end{array}\right)=0$ that holds for any integer $n>0$.

We can now easily derive Theorem 2.10 from Theorem 2.11:

Proof of Theorem 2.10: We have

$$
\sum_{F \subseteq E}(-1)^{|F|}=\sum_{\substack{F \subseteq E ; \\ G \subseteq \text { Shade } F}}(-1)^{|F|}+\underbrace{\sum_{\substack{F \subseteq E ; \\ \text { shade } F}}(-1)^{|F|}}_{\substack{=0 \\ \text { (by Theorem 2.11 }}}=\sum_{\substack{F \subseteq E ; \\ G \subseteq \text { Shade } F}}(-1)^{|F|} .
$$

However, Lemma 2.12 (applied to $U=E$ ) shows that the left hand side of this equality is 0 . Thus, so is the right hand side. This proves Theorem 2.10 .

\subsection{Proving Theorem 1.2}

Proof of Theorem 1.2: Theorem 1.2 follows by applying Theorem 2.10 to $G=E$ (since a subset $F$ of $E$ satisfies $E \subseteq$ Shade $F$ if and only if it is pandemic).

\section{Vertex infection and other variants}

In our study of graphs so far, we have barely ever mentioned vertices (even though they are, of course, implicit in the notion of a path). Even though the infection is spread from vertex to vertex, our sets so far 
have infected edges (not vertices). One might thus wonder if there is also a vertex counterpart of Theorem 1.2. So let us define analogues of our notions for vertices:

If $F \subseteq V$, then an $F$-vertex-path shall mean a path of $\Gamma$ such that all vertices of the path except (possibly) for its two endpoints belong to $F$. (Thus, if a path has only one edge or none, then it automatically is an $F$-vertex-path.)

If $w \in V \backslash\{v\}$ is any vertex and $F \subseteq V \backslash\{v\}$ is any subset, then we say that $F$ vertex-infects $w$ if there exists an $F$-vertex-path from $v$ to $w$. (This is always true when $w$ is $v$ or a neighbor of $v$.)

A subset $F \subseteq V \backslash\{v\}$ is said to be vertex-pandemic if it vertex-infects each vertex $w \in V \backslash\{v\}$.

Example 3.1. Let $\Gamma$ be as in Example 1.3. Then, the path $v \stackrel{1}{\longrightarrow} p \stackrel{2}{\longrightarrow} q$ is an $F$-vertex-path for any subset $F \subseteq V$ that satisfies $p \in F$. The subset $\{p\}$ of $V \backslash\{v\}$ vertex-infects each vertex (for example, $v \stackrel{1}{\longrightarrow} p \stackrel{2}{\longrightarrow} q$ is a $\{p\}$-vertex-path from $v$ to $q$, and $v \stackrel{4}{\longrightarrow} w$ is a $\{p\}$-vertex-path from $v$ to $w$ ), and thus is vertex-pandemic. The vertex-pandemic subsets of $V \backslash\{v\}$ are the sets

$$
\{p\},\{w\},\{p, q\},\{p, w\},\{q, w\},\{p, q, w\} .
$$

We now have the following analogue of Theorem 1.2:

Theorem 3.2. Assume that $V \backslash\{v\} \neq \varnothing$. Then,

$$
\sum_{\substack{F \subseteq V \backslash\{v\} \text { is } \\ \text { vertex-pandemic }}}(-1)^{|F|}=0 .
$$

Proof: With a few easy modifications, our above proof of Theorem 1.2 can be repurposed as a proof of Theorem 3.2. Most importantly, we need to replace the set $E$ by $V \backslash\{v\}$, and we need to replace the words "edge", "F-path", "infects" and "pandemic" by "vertex", " $F$-vertex-path", "vertex-infects" and "vertex-pandemic", respectively.

Another variant of Theorem 1.2 (and Theorem 2.10 and Theorem 2.11) is obtained by replacing the undirected graph $\Gamma$ with a directed graph (while, of course, replacing paths by directed paths). More generally, we can replace $\Gamma$ by a "hybrid" graph with some directed and some undirected edges. (ii) No changes are required to the above proofs. Yet another variation can be obtained by replacing "endpoint" by "source" (for directed edges). We cannot, however, replace "endpoint" by "target".

\section{An abstract perspective}

Seeing how little graph theory we have used in proving Theorem 1.2, and how easily the same argument adapted to Theorem 3.2, we get the impression that there might be some general theory lurking behind it. What follows is an attempt at building this theory.

Most proofs in this section are omitted; some are outlined. In fact, they are all sufficiently simple and straightforward that the reader should have little trouble filling them in; alternatively, almost all of them can be found in the detailed version of Grinberg (2021).

(ii) We understand that a directed edge still has two endpoints: its source and its target. 


\subsection{Shade maps}

Let $\mathcal{P}(E)$ denote the power set of $E$. In Definition 2.6, we have encoded the "infects" relation as a map Shade : $\mathcal{P}(E) \rightarrow \mathcal{P}(E)$ defined by Shade $F=\{e \in E \mid F$ infects $e\}$. As we recall, Theorem 2.10 (a generalization of Theorem 1.2 states that

$$
\sum_{\substack{F \subseteq E ; \\ G \subseteq \text { Shade } F}}(-1)^{|F|}=0
$$

for any $G \subseteq E$, under the assumption that $E \neq \varnothing$.

To generalize this, we forget about the graph $\Gamma$ and the map Shade, and instead start with an arbitrary finite set $E$. (This set $E$ corresponds to the set $E$ in Theorem 1.2 and to the set $V \backslash\{v\}$ in Theorem 3.2.) Let $\mathcal{P}(E)$ be the power set of $E$. Let Shade $: \mathcal{P}(E) \rightarrow \mathcal{P}(E)$ be an arbitrary map (meant to generalize the map Shade from the previous paragraph). We may now ask:

Question 4.1. What (combinatorial) properties must Shade satisfy in order for (9) to hold for any $G \subseteq E$ under the assumption that $E \neq \varnothing$ ?

A partial answer to this question can be given by analyzing our above proof of Theorem 2.10 and extracting what was used:

Definition 4.2. Let $E$ be a set. A shade map on $E$ shall mean a map Shade $: \mathcal{P}(E) \rightarrow \mathcal{P}(E)$ that satisfies the following two axioms:

Axiom 1: If $F \in \mathcal{P}(E)$ and $u \in E \backslash$ Shade $F$, then Shade $(F \cup\{u\})=\operatorname{Shade} F$.

Axiom 2: If $F \in \mathcal{P}(E)$ and $u \in E \backslash$ Shade $F$, then Shade $(F \backslash\{u\})=$ Shade $F$.

Theorem 4.3. Let $E$ be a finite set. Let Shade $: \mathcal{P}(E) \rightarrow \mathcal{P}(E)$ be a shade map on $E$.

Assume that $E \neq \varnothing$. Let $G$ be any subset of $E$. Then,

$$
\sum_{\substack{F \subseteq E ; \\ G \subseteq \text { Shade } F}}(-1)^{|F|}=0 .
$$

Proof: Again, the proof is analogous to our above proof of Theorem 2.10. (This time, in the proof of Lemma 2.9, the equalities (5) and (6) follow directly from Axiom 1 and Axiom 2, respectively.)

How do shade maps relate to known concepts in the combinatorics of set families (such as topologies, clutters, matroids, or submodular functions)? Are they just one of these known concepts in disguise? We shall answer two versions of this question in the following subsections. Specifically:

- In Subsection 4.3, we will show that inclusion-reversing shade maps on $E$ (i.e., shade maps Shade that satisfy Shade $B \subseteq$ Shade $A$ whenever $A \subseteq B$ ) are in bijection with antimatroidal quasiclosure operators (a slight variant of antimatroids) on $E$.

- In Subsection 4.4, we will show that arbitrary shade maps are in bijection with Boolean interval partitions of $\mathcal{P}(E)$ (that is, set partitions of $\mathcal{P}(E)$ into intervals of the Boolean lattice $\mathcal{P}(E)$ ). 
Before we come to these characterizations, we shall however make a few elementary remarks on shade maps.

First, we observe that Axioms 1 and 2 in Definition 4.2 can be weakened to the following statements:

Axiom 1': If $F \in \mathcal{P}(E)$ and $u \in E \backslash$ Shade $F$, then Shade $(F \cup\{u\}) \subseteq$ Shade $F$.

Axiom 2': If $F \in \mathcal{P}(E)$ and $u \in E \backslash$ Shade $F$, then Shade $(F \backslash\{u\}) \subseteq$ Shade $F$.

Axiom 1' is weaker than Axiom 1, and likewise Axiom 2' is weaker than Axiom 2. However, Axioms 1 ' and 2' combined are equivalent to Axioms 1 and 2 combined:

Proposition 4.4. Let $E$ be a set. Let Shade $: \mathcal{P}(E) \rightarrow \mathcal{P}(E)$ be any map. Then, Shade is a shade map on $E$ if and only if Shade satisfies the two Axioms 1' and 2' stated above.

Axioms 1 and 2 can also be combined into one common axiom:

Axiom 3: If $F \in \mathcal{P}(E)$ and $u \in E \backslash F$, then we have Shade $F=\operatorname{Shade}(F \cup\{u\})$ or $u \in($ Shade $F) \cap \operatorname{Shade}(F \cup\{u\})$.

Proposition 4.5. Let $E$ be a set. Let Shade $: \mathcal{P}(E) \rightarrow \mathcal{P}(E)$ be any map. Then, Shade is a shade map on $E$ if and only if Shade satisfies Axiom 3.

We will soon see some examples. First, let us introduce two more basic concepts that will help clarify these examples:

Definition 4.6. Let $E$ be a set. Let Shade $: \mathcal{P}(E) \rightarrow \mathcal{P}(E)$ be any map (not necessarily a shade map).

(a) We say that Shade is inclusion-preserving if it satisfies the following property: If $A$ and $B$ are two subsets of $E$ such that $A \subseteq B$, then Shade $A \subseteq$ Shade $B$.

(b) We say that Shade is inclusion-reversing if it satisfies the following property: If $A$ and $B$ are two subsets of $E$ such that $A \subseteq B$, then Shade $B \subseteq$ Shade $A$.

For instance, the map Shade from Definition 2.6 is inclusion-preserving (because of Lemma 2.8) and is a shade map (by Lemma 2.9). The same holds for the analogue of the map Shade that uses vertexinfection instead of infection. We will soon see some inclusion-reversing shade maps, and it is not hard to construct shade maps that are neither inclusion-preserving nor inclusion-reversing.

Let us observe that there is a simple bijection between inclusion-preserving and inclusion-reversing maps, and this bijection preserves shadeness:

Proposition 4.7. Let $E$ be a set. Let Shade $: \mathcal{P}(E) \rightarrow \mathcal{P}(E)$ be any map (not necessarily a shade map). Let Shade $^{\prime}: \mathcal{P}(E) \rightarrow \mathcal{P}(E)$ be the map that sends each $F \in \mathcal{P}(E)$ to Shade $(E \backslash F) \in \mathcal{P}(E)$. Then:

(a) The map Shade is inclusion-preserving if and only if the map Shade' ${ }^{\prime}$ is inclusion-reversing.

(b) The map Shade is a shade map if and only if the map Shade' is a shade map.

Definition 4.8. Let $E$, Shade and Shade' be as in Proposition 4.7. We then say that the map Shade' is dual to Shade. 


\subsection{Some examples of shade maps}

As we already mentioned, Lemma 2.9 and its analogue for vertex-infection provide two examples of inclusion-preserving shade maps Shade. An example of an inclusion-reversing shade map comes from the theory of posets:

Example 4.9. Let $E$ be a poset. For any $F \subseteq E$, we define

$$
F_{\downarrow}=\{e \in E \mid \text { there exists an } f \in F \text { with } e<f\}
$$

and

$$
\text { Shade } F=E \backslash F_{\downarrow} \text {. }
$$

Then, this map Shade $: \mathcal{P}(E) \rightarrow \mathcal{P}(E)$ is an inclusion-reversing shade map.

Another example of a shade map comes from discrete geometry:

Example 4.10. Let $A$ be an affine space over $\mathbb{R}$. If $S$ is a finite subset of $A$, then a nontrivial convex combination of $S$ will mean a point of the form $\sum_{s \in S} \lambda_{s} s \in A$, where the coefficients $\lambda_{s}$ are nonnegative reals smaller than 1 and satisfying $\sum_{s \in S} \lambda_{s}=1$.

Fix a finite subset $E$ of $A$. For any $F \subseteq E$, we define

$$
\text { Shade } F=\{e \in E \mid e \text { is not a nontrivial convex combination of } F\} \text {. }
$$

Then, this map Shade $: \mathcal{P}(E) \rightarrow \mathcal{P}(E)$ is an inclusion-reversing shade map.

As a contrast to Example 4.10, let us mention a not-quite-example (satisfying only one of the two axioms in Theorem 4.3):

Example 4.11. Let $V$ be a vector space over $\mathbb{R}$. If $S$ is a finite subset of $V$, then a nontrivial conic combination of $S$ will mean a vector of the form $\sum_{s \in S} \lambda_{s} s \in V$, where the coefficients $\lambda_{s}$ are nonnegative reals with the property that at least two elements $s \in S$ satisfy $\lambda_{s}>0$.

Fix a finite subset $E$ of $V$. For any $F \subseteq E$, we define

$$
\text { Shade } F=\{e \in E \mid e \text { is not a nontrivial conic combination of } F\} \text {. }
$$

It can be shown that this map Shade $: \mathcal{P}(E) \rightarrow \mathcal{P}(E)$ satisfies Axiom 1 in Definition 4.2. In general, it does not satisfy Axiom 2 . Thus, it is not a shade map in general.

\subsection{Antimatroids and inclusion-reversing shade maps}

Examples 4.9 and 4.10 are instances of a general class of examples: shade maps coming from antimatroids. Not unlike matroids, antimatroids are a combinatorial concept with many equivalent avatars (see, e.g., Korte et al, 1991, Chapter III)). Here we shall view them through one of these avatars: that of antimatroidal quasi-closure operators (roughly equivalent to convex geometries). We begin by defining the notions we need:

Definition 4.12. Let $E$ be any set.

(a) A quasi-closure operator on $E$ means a map $\tau: \mathcal{P}(E) \rightarrow \mathcal{P}(E)$ with the following properties:

1. We have $A \subseteq \tau(A)$ for any $A \subseteq E$. 
2. If $A$ and $B$ are two subsets of $E$ satisfying $A \subseteq B$, then $\tau(A) \subseteq \tau(B)$.

3. We have $\tau(\tau(A))=\tau(A)$ for any $A \subseteq E$.

(b) A quasi-closure operator $\tau$ on $E$ is said to be antimatroidal if it has the following additional property:

4. If $X$ is a subset of $E$, and if $y$ and $z$ are two distinct elements of $E \backslash \tau(X)$ satisfying $z \in$ $\tau(X \cup\{y\})$, then $y \notin \tau(X \cup\{z\})$.

(c) A closure operator on $E$ means a quasi-closure operator $\tau$ on $E$ that satisfies $\tau(\varnothing)=\varnothing$.

(d) If $\tau$ is an antimatroidal closure operator on $E$, then the pair $(E, \tau)$ is called a convex geometry.

Here are some examples of antimatroidal quasi-closure operators:

Example 4.13. Let $E$ be a poset. For any $F \subseteq E$, we define

$$
\tau(F)=\{e \in E \mid \text { there exists an } f \in F \text { with } e \leq f\} .
$$

Then, $\tau$ is an antimatroidal closure operator on $E$. (This example is the "downset alignment" from (Edelman and Jamison, 1985, §3, Example II), and is equivalent to the "poset antimatroid" from (Korte et al. 1991, §III.2.3).)

Example 4.14. Let $E$ be a poset. For any $F \subseteq E$, we define

$$
\tau(F)=\{e \in E \mid \text { there exist } f \in F \text { and } g \in F \text { with } g \leq e \leq f\} .
$$

Then, $\tau$ is an antimatroidal closure operator on $E$. (This example is the "order convex alignment" from Edelman and Jamison, 1985, §3, Example II), and is the "double shelling of a poset" example from (Korte et al., 1991, §III.2.4).)

Example 4.15. Let $A$ be an affine space over $\mathbb{R}$. If $S$ is a finite subset of $A$, then a convex combination of $S$ will mean a point of the form $\sum_{s \in S} \lambda_{s} s \in A$, where the coefficients $\lambda_{s}$ are nonnegative reals satisfying $\sum_{s \in S} \lambda_{s}=1$.

Fix a finite subset $E$ of $A$. For any $F \subseteq E$, we define

$$
\tau(F)=\{e \in E \mid e \text { is a convex combination of } F\} .
$$

Then, $\tau$ is an antimatroidal closure operator on $E$. (This example is (Edelman and Jamison, 1985, $\$ 3$, Example I); it gave the name "convex geometry" to the notion defined in Definition 4.12 (d).)

Example 4.16. Let $\Gamma$ be any graph with edge set $E$. Fix a vertex $v$ of $\Gamma$. We say that a subset $F \subseteq E$ blocks an edge $e \in E$ if each path of $\Gamma$ that contains $v$ and $e$ must contain at least one edge of $F$. (In particular, this is automatically the case when $e \in F$.) For each $F \subseteq E$, we define

$$
\tau(F)=\{e \in E \mid F \text { blocks } e\} .
$$

Then, $\tau$ is an antimatroidal quasi-closure operator on $E$. (This example is the "line-search antimatroid" from (Korte et al., 1991, §III.2.11).) If $\Gamma$ is connected, then $\tau$ is actually a closure operator. 
Further examples of antimatroidal closure operators can be found in (Korte et al., 1991, §III.2) and (Edelman and Jamison, 1985, §3).

We shall be dealing with quasi-closure operators rather than closure operators most of the time. However, since the latter concept is somewhat more widespread, let us comment on the connection between the two. Roughly speaking, the relation between quasi-closure and closure operators is comparable to the relation between semigroups and monoids, or between nonunital rings and unital rings, or (perhaps the best analogue) between simplicial complexes in general and simplicial complexes without ghost vertices (i.e., simplicial complexes for which every element of the ground set is a dimension- 0 face). More concretely, specifying a quasi-closure operator on a set $E$ is tantamount to specifying a subset of $E$ and a closure operator on this subset. To wit:

Proposition 4.17. Let $E$ be a set. Let $L$ be a subset of $E$.

(a) Then, there is a bijection

$$
\begin{aligned}
& \text { from }\{\text { quasi-closure operators } \tau \text { on } E \text { satisfying } \tau(\varnothing)=L\} \\
& \text { to }\{\text { closure operators } \sigma \text { on } E \backslash L\}
\end{aligned}
$$

that is defined as follows: It sends each quasi-closure operator $\tau$ to the closure operator $\sigma$ that sends each $F \subseteq E \backslash L$ to $\tau(F) \backslash L$.

(b) This bijection restricts to a bijection

$$
\begin{aligned}
& \text { from }\{\text { antimatroidal quasi-closure operators } \tau \text { on } E \text { satisfying } \tau(\varnothing)=L\} \\
& \text { to }\{\text { antimatroidal closure operators } \sigma \text { on } E \backslash L\}
\end{aligned}
$$

Now, we claim the following:

Theorem 4.18. Let $E$ be a set. Let $\tau: \mathcal{P}(E) \rightarrow \mathcal{P}(E)$ be an antimatroidal quasi-closure operator on $E$. For any $F \subseteq E$, we define

$$
\text { Shade } F=\{e \in E \mid e \notin \tau(F \backslash\{e\})\} .
$$

Then, this map Shade $: \mathcal{P}(E) \rightarrow \mathcal{P}(E)$ is an inclusion-reversing shade map.

Theorem 4.18 generalizes Examples 4.9 and 4.10. Indeed, the latter two examples are obtained by applying Theorem 4.18 to the settings of Examples 4.13 and 4.15 , respectively. Less directly, Lemma 2.9 and its vertex-infection analogue are particular cases of Theorem 4.18 as well (even though they involve shade maps that are inclusion-preserving rather than inclusion-reversing). Indeed, if we apply Theorem 4.18 to the setting of Example 4.16, then we obtain the claim of Lemma 2.9 with Shade $F$ replaced by Shade $(E \backslash F)$; this is easily seen to be equivalent to Lemma 2.9 (by the duality stated in Proposition 4.7).

Our proof of Theorem 4.18 will rely on two easy lemmas:

Lemma 4.19. Let $E$ be a set. Let $\tau$ be a quasi-closure operator on $E$. Let $X$ be a subset of $E$, and let $z \in \tau(X)$. Then, $\tau(X \cup\{z\})=\tau(X)$.

Lemma 4.20. Let $E$ be a set. Let $\tau$ be an antimatroidal quasi-closure operator on $E$. Let $X$ be a subset of $E$, and let $y$ and $z$ be two distinct elements of $E$ satisfying $z \in \tau(X \cup\{y\})$ and $y \in \tau(X \cup\{z\})$. Then, $y \in \tau(X)$. 
Note that Lemma 4.20 has a converse: If $\tau$ is a quasi-closure operator on $E$ satisfying the claim of Lemma 4.20, then $\tau$ is antimatroidal. This is easy to see but will not be used in what follows.

Proof of Theorem 4.18: We shall prove the following three statements:

Statement 0: If $A$ and $B$ are two subsets of $E$ such that $A \subseteq B$, then Shade $B \subseteq$ Shade $A$.

Statement 1: If $F \in \mathcal{P}(E)$ and $u \in E \backslash$ Shade $F$, then Shade $(F \cup\{u\})=$ Shade $F$.

Statement 2: If $F \in \mathcal{P}(E)$ and $u \in E \backslash$ Shade $F$, then Shade $(F \backslash\{u\})=$ Shade $F$.

Our proofs of these three statements will use Property 2 in Definition 4.12 (a) (which we shall just refer to as "Property 2").

[Proof of Statement 0: Let $A$ and $B$ be two subsets of $E$ such that $A \subseteq B$. We must prove that Shade $B \subseteq$ Shade $A$.

Let $u \in$ Shade $B$. Thus, $u \in E$ and $u \notin \tau(B \backslash\{u\})$ (by the definition of Shade $B$ ). However, $A \backslash\{u\} \subseteq B \backslash\{u\}$ (since $A \subseteq B$ ) and thus $\tau(A \backslash\{u\}) \subseteq \tau(B \backslash\{u\})$ (by Property 2). Hence, from $u \notin \tau(B \backslash\{u\})$, we obtain $u \notin \tau(A \backslash\{u\})$. Therefore, $u \in$ Shade $A$ (by the definition of Shade $A$ ).

Since we have shown this for each $u \in$ Shade $B$, we thus obtain Shade $B \subseteq$ Shade $A$. This proves Statement 0.]

[Proof of Statement 2: Let $F \in \mathcal{P}(E)$ and $u \in E \backslash$ Shade $F$. We must prove that Shade $(F \backslash\{u\})=$ Shade $F$.

We have $u \in E \backslash$ Shade $F$, so that $u \notin$ Shade $F$. In other words, $u \in E$ and $u \in \tau(F \backslash\{u\})$ (by the definition of Shade $F$ ).

We have $F \backslash\{u\} \subseteq F$ and thus Shade $F \subseteq$ Shade $(F \backslash\{u\})$ (by Statement 0).

Now, let $v \in$ Shade $(F \backslash\{u\})$. We shall prove that $v \in$ Shade $F$.

Indeed, assume the contrary. Hence, $v \notin$ Shade $F$. In other words, $v \in E$ and $v \in \tau(F \backslash\{v\})$ (by the definition of Shade $F$ ).

Let $X=(F \backslash\{u\}) \backslash\{v\}$. Then, $F \backslash\{u\} \subseteq X \cup\{v\}$ and therefore $\tau(F \backslash\{u\}) \subseteq \tau(X \cup\{v\})$ (by Property 2). Hence, $u \in \tau(F \backslash\{u\}) \subseteq \tau(X \cup\{v\})$.

Also, from $X=(F \backslash\{u\}) \backslash\{v\}=(F \backslash\{v\}) \backslash\{u\}$, we obtain $F \backslash\{v\} \subseteq X \cup\{u\}$ and therefore $\tau(F \backslash\{v\}) \subseteq \tau(X \cup\{u\})$ (by Property 2). Hence, $v \in \tau(F \backslash\{v\}) \subseteq \tau(X \cup\{u\})$.

However, from $v \in$ Shade $(F \backslash\{u\})$, we obtain $v \notin \tau((F \backslash\{u\}) \backslash\{v\})$ (by the definition of Shade $(F \backslash\{u\})$ ). In other words, $v \notin \tau(X)$ (since $X=(F \backslash\{u\}) \backslash\{v\}$ ). However, if $v$ and $u$ were distinct, then Lemma 4.20 (applied to $y=v$ and $z=u$ ) would yield $v \in \tau(X)$ (since $v \in \tau(X \cup\{u\})$ and $u \in \tau(X \cup\{v\})$ ), which would contradict $v \notin \tau(X)$. Thus, $v$ and $u$ cannot be distinct. In other words, $v=u$. Now, $X=(F \backslash\{u\}) \backslash\{v\}=F \backslash\{u\}$ (since $v=u$ ). Therefore, $u \in \tau(F \backslash\{u\})$ rewrites as $u \in \tau(X)$. Hence, $v=u \in \tau(X)$; but this contradicts $v \notin \tau(X)$.

This contradiction shows that our assumption was false; hence, we conclude that $v \in$ Shade $F$. Since we have proved this for each $v \in \operatorname{Shade}(F \backslash\{u\})$, we thus obtain Shade $(F \backslash\{u\}) \subseteq$ Shade $F$. Combining this with Shade $F \subseteq$ Shade $(F \backslash\{u\})$, we obtain Shade $(F \backslash\{u\})=$ Shade $F$. This proves Statement 2.]

[Proof of Statement 1: Let $F \in \mathcal{P}(E)$ and $u \in E \backslash$ Shade $F$. We must prove that Shade $(F \cup\{u\})=$ Shade $F$. If $u \in F$, then this is obvious (since $F \cup\{u\}=F$ in this case). Thus, we WLOG assume that $u \notin F$. Hence, $(F \cup\{u\}) \backslash\{u\}=F$. 
We have $F \subseteq F \cup\{u\}$ and thus Shade $(F \cup\{u\}) \subseteq$ Shade $F$ (by Statement 0).

Now, $u \in E \backslash$ Shade $F \subseteq E \backslash$ Shade $(F \cup\{u\})$ (since Shade $(F \cup\{u\}) \subseteq$ Shade $F$ ). Hence, Statement 2 (applied to $F \cup\{u\}$ instead of $F$ ) yields Shade $F=$ Shade $(F \cup\{u\}$ ) (since $(F \cup\{u\}) \backslash$ $\{u\}=F)$. This proves Statement 1.]

Now, we have proved Statements 1 and 2. Thus, the map Shade $: \mathcal{P}(E) \rightarrow \mathcal{P}(E)$ is a shade map. Moreover, this map is inclusion-reversing (by Statement 0). Thus, Theorem 4.18 is proved.

We note that the quasi-closure operator $\tau$ in Theorem 4.18 can be reconstructed from the map Shade. This does not even require $\tau$ to be antimatroidal; the following holds for any quasi-closure operator:

Proposition 4.21. Let $E$ be a set. Let $\tau: \mathcal{P}(E) \rightarrow \mathcal{P}(E)$ be a quasi-closure operator on E. For any $F \subseteq E$, we define

$$
\text { Shade } F=\{e \in E \mid e \notin \tau(F \backslash\{e\})\} \text {. }
$$

Then, each $F \subseteq E$ satisfies

$$
\tau(F)=F \cup(E \backslash \text { Shade } F) .
$$

It turns out that if one applies the formula (11) to an inclusion-reversing shade map Shade, then the resulting map $\tau$ is an antimatroidal quasi-closure operator, at least when $E$ is finite. In fact, we have the following:

Proposition 4.22. Let $E$ be a finite set. Let Shade $: \mathcal{P}(E) \rightarrow \mathcal{P}(E)$ be an inclusion-reversing shade map. Define a map $\tau: \mathcal{P}(E) \rightarrow \mathcal{P}(E)$ by setting

$$
\tau(F)=F \cup(E \backslash \text { Shade } F)
$$

for each $F \subseteq E$. Then, $\tau$ is an antimatroidal quasi-closure operator on $E$.

The proof of this proposition rests on the following lemma:

Lemma 4.23. Let $E$ be a set. Let Shade $: \mathcal{P}(E) \rightarrow \mathcal{P}(E)$ be a shade map. Let $A$ and $B$ be two subsets of $E$ such that $B$ is finite and $B \cap$ Shade $A=\varnothing$. Then, Shade $(A \cup B)=$ Shade $A$.

Proposition 4.21 has a (sort of) converse:

Proposition 4.24. Let $E$ be a set. Let Shade $: \mathcal{P}(E) \rightarrow \mathcal{P}(E)$ be an inclusion-reversing shade map. For any $F \subseteq E$, we define

$$
\tau(F)=F \cup(E \backslash \text { Shade } F) .
$$

Then, each $F \subseteq E$ satisfies

$$
\text { Shade } F=\{e \in E \mid e \notin \tau(F \backslash\{e\})\} .
$$

Combining many of the results in this section, we obtain the following description of inclusion-reversing shade maps:

Theorem 4.25. Let $E$ be a finite set. Then, there is a bijection

$$
\begin{aligned}
& \text { from the set }\{\text { inclusion-reversing shade maps Shade }: \mathcal{P}(E) \rightarrow \mathcal{P}(E)\} \\
& \text { to the set }\{\text { antimatroidal quasi-closure operators } \tau: \mathcal{P}(E) \rightarrow \mathcal{P}(E)\}
\end{aligned}
$$

It sends each map Shade to the map $\tau$ defined by (12). Its inverse map sends each map $\tau$ to the map Shade defined by (10). 
Theorem 4.25 classifies inclusion-reversing shade maps in terms of antimatroidal quasi-closure operators (iii). The latter can in turn be described in terms of antimatroidal closure operators (by Proposition 4.17), i.e., in terms of antimatroids. Thus, inclusion-reversing shade maps "boil down" to antimatroids. The same can be said of inclusion-preserving shade maps (because Proposition 4.7 establishes a bijection between them and the inclusion-reversing ones). In the next subsection, we shall classify arbitrary shade maps in terms of what we will call Boolean interval partitions.

\subsection{Boolean interval partitions and arbitrary shade maps}

Let us first define Boolean interval partitions:

Definition 4.26. Let $E$ be a set.

(a) If $U$ and $V$ are two subsets of $E$ satisfying $U \subseteq V$, then $[U, V]$ shall denote the subset

$$
\{I \in \mathcal{P}(E) \mid U \subseteq I \subseteq V\}
$$

of $\mathcal{P}(E)$. This is the set of all subsets of $E$ that lie between $U$ and $V$ (meaning that they contain $U$ as a subset, but in turn are contained in $V$ as subsets).

(b) A Boolean interval of $\mathcal{P}(E)$ shall mean a subset of $\mathcal{P}(E)$ that has the form $[U, V]$ for two subsets $U$ and $V$ of $E$ satisfying $U \subseteq V$. Note that each Boolean interval $[U, V]$ of $\mathcal{P}(E)$ is nonempty (as it contains $U$ and $V$ ), and the two subsets $U$ and $V$ can easily be reconstructed from it (namely, $U$ is the intersection of all $I \in[U, V]$, whereas $V$ is the union of all $I \in[U, V]$ ).

(c) A Boolean interval partition of $\mathcal{P}(E)$ means a set of pairwise disjoint Boolean intervals of $\mathcal{P}(E)$ whose union is $\mathcal{P}(E)$.

(d) If $\mathbf{P}$ is a Boolean interval partition of $\mathcal{P}(E)$, then the elements of $\mathbf{P}$ (that is, the Boolean intervals that belong to $\mathbf{P}$ ) are called the blocks of $\mathbf{P}$.

Example 4.27. For this example, let $E=\{1,2,3\}$. We shall use the shorthand $i_{1} i_{2} \cdots i_{k}$ for a subset $\left\{i_{1}, i_{2}, \ldots, i_{k}\right\}$ of $E$. (For example, 13 means the subset $\{1,3\}$.)

(a) We have

$$
[1,123]=\{I \in \mathcal{P}(123) \mid 1 \subseteq I \subseteq 123\}=\{1,12,13,123\}
$$

and

$$
[1,13]=\{I \in \mathcal{P}(123) \mid 1 \subseteq I \subseteq 13\}=\{1,13\}
$$

(b) There are $3^{3}=27$ Boolean intervals of $\mathcal{P}(E)$. (More generally, if $E$ is an $n$-element set, then there are $3^{n}$ Boolean intervals of $\mathcal{P}(E)$.)

(c) Here is one of many Boolean interval partitions of $\mathcal{P}(E)$ (where $E$ is still $\{1,2,3\}$ ):

$$
\{\underbrace{\{\varnothing\}}_{=[\varnothing, \varnothing]}, \underbrace{\{1,13\}}_{=[1,13]}, \underbrace{\{3\}}_{=[3,3]}, \underbrace{\{2,12,23,123\}}_{=[2,123]}\} .
$$

Here is another:

$$
\{\underbrace{\{\varnothing, 1\}}_{=[\varnothing, 1]}, \underbrace{\{3,13\}}_{=[3,13]}, \underbrace{\{2,23\}}_{=[2,23]}, \underbrace{\{12\}}_{=[12,12]}, \underbrace{\{123\}}_{=[123,123]}\} .
$$

(iii) In the parlance of matroid theorists, it shows that inclusion-reversing shade maps are cryptomorphic to antimatroidal quasiclosure operators. 
The former has four blocks; the latter has five.

Here are two ways to think of Boolean interval partitions of $\mathcal{P}(E)$ :

- The following is just a slick restatement of Definition 4.26 (c) using standard combinatorial lingo: A Boolean interval partition of $\mathcal{P}(E)$ is a set partition of the Boolean lattice $\mathcal{P}(E)$ into intervals.

- It is well-known that the set partitions of a given set are in a canonical bijection with the equivalence relations on this set. In light of this, the Boolean interval partitions of $\mathcal{P}(E)$ can be viewed as the equivalence relations on $\mathcal{P}(E)$ whose equivalence classes are Boolean intervals. In other words, they can be viewed as the equivalence relations $\sim$ on $\mathcal{P}(E)$ satisfying the axiom "if $U, V, I \in \mathcal{P}(E)$ satisfy $U \sim V$ and $U \cap V \subseteq I \subseteq U \cup V$, then $U \sim I \sim V$ ". The reader can prove this alternative characterization as an easy exercise in Boolean algebra.

Boolean interval partitions have come up in combinatorics before (e.g., Brunat et al. (2009), Dawson 1981), Dedndreaj and Tittmann (2021), Gordon and McMahon (1997).

We shall now construct a shade map from any Boolean interval partition:

Theorem 4.28. Let $E$ be a set. Let $\mathbf{P}$ be a Boolean interval partition of $\mathcal{P}(E)$.

For any $F \in \mathcal{P}(E)$, let $[\alpha(F), \tau(F)]$ denote the (unique) block of $\mathbf{P}$ that contains $F$.

We define a map Shade $: \mathcal{P}(E) \rightarrow \mathcal{P}(E)$ by setting

$$
\text { Shade } F=E \backslash(\tau(F) \backslash \alpha(F)) \quad \text { for any } F \in \mathcal{P}(E) .
$$

Then:

(a) The map Shade is a shade map on $E$.

(b) We have $\alpha(F)=F \cap$ Shade $F$ and $\tau(F)=F \cup(E \backslash$ Shade $F)$ for any $F \in \mathcal{P}(E)$.

(c) We have $\mathbf{P}=\{[\alpha(F), \tau(F)] \mid F \in \mathcal{P}(E)\}$.

The proof of Theorem 4.28 is rather easy. We lighten our burden somewhat with a simple lemma (which can be easily checked using Venn diagrams):

Lemma 4.29. Let $X, Y, Z$ and $E$ be four sets such that $X \subseteq Y \subseteq Z \subseteq E$. Then:

(a) We have $Y \cup(Z \backslash X)=Z$.

(b) We have $Y \cap(E \backslash(Z \backslash X))=X$.

Proof of Theorem 4.28: (a) We shall prove the following two statements:

Statement 1: If $F \in \mathcal{P}(E)$ and $u \in E \backslash$ Shade $F$, then Shade $(F \cup\{u\})=$ Shade $F$.

Statement 2: If $F \in \mathcal{P}(E)$ and $u \in E \backslash$ Shade $F$, then Shade $(F \backslash\{u\})=$ Shade $F$.

[Proof of Statement 1: Let $F \in \mathcal{P}(E)$ and $u \in E \backslash$ Shade $F$. We must prove that Shade $(F \cup\{u\})=$ Shade $F$.

The definition of $\alpha(F)$ and $\tau(F)$ reveals that $[\alpha(F), \tau(F)]$ is the (unique) block of $\mathbf{P}$ that contains $F$. Thus, $[\alpha(F), \tau(F)]$ is a block of $\mathbf{P}$ and contains $F$.

The definition of Shade yields Shade $F=E \backslash(\tau(F) \backslash \alpha(F))$. Thus, $E \backslash$ Shade $F=\tau(F) \backslash \alpha(F)$ (since $\tau(F) \backslash \alpha(F)$ is a subset of $E$ ). Now, $u \in E \backslash$ Shade $F=\tau(F) \backslash \alpha(F)$. In other words, $u \in \tau(F)$ and $u \notin \alpha(F)$. 
On the other hand, the Boolean interval $[\alpha(F), \tau(F)]$ contains $F$. In other words, $\alpha(F) \subseteq F \subseteq$ $\tau(F)$.

Now, set $F^{\prime}=F \cup\{u\}$. From $F \subseteq \tau(F)$ and $u \in \tau(F)$, we thus obtain $F^{\prime} \subseteq \tau(F)$. Combined with $\alpha(F) \subseteq F \subseteq F^{\prime}$, this entails $F^{\prime} \in[\alpha(F), \tau(F)]$. Hence, $[\alpha(F), \tau(F)]$ is a block of $\mathbf{P}$ that contains $F^{\prime}$ (since we already know that $[\alpha(F), \tau(F)]$ is a block of $\mathbf{P}$ ).

However, the definition of $\alpha\left(F^{\prime}\right)$ and $\tau\left(F^{\prime}\right)$ shows that $\left[\alpha\left(F^{\prime}\right), \tau\left(F^{\prime}\right)\right]$ is the (unique) block of $\mathbf{P}$ that contains $F^{\prime}$. Since we know that $[\alpha(F), \tau(F)]$ is a block of $\mathbf{P}$ that contains $F^{\prime}$, we therefore conclude that $\left[\alpha\left(F^{\prime}\right), \tau\left(F^{\prime}\right)\right]=[\alpha(F), \tau(F)]$. Hence, we have

$$
\alpha\left(F^{\prime}\right)=\alpha(F) \quad \text { and } \quad \tau\left(F^{\prime}\right)=\tau(F)
$$

(because a Boolean interval $[U, V]$ uniquely determines both $U$ and $V$ ). Now, the definition of Shade yields

$$
\text { Shade }\left(F^{\prime}\right)=E \backslash(\underbrace{\tau\left(F^{\prime}\right)}_{=\tau(F)} \backslash \underbrace{\alpha\left(F^{\prime}\right)}_{=\alpha(F)})=E \backslash(\tau(F) \backslash \alpha(F))=\text { Shade } F \text {. }
$$

In view of $F^{\prime}=F \cup\{u\}$, this rewrites as Shade $(F \cup\{u\})=$ Shade $F$. This proves Statement 1.]

[Proof of Statement 2: Let $F \in \mathcal{P}(E)$ and $u \in E \backslash$ Shade $F$. We must prove that Shade $(F \backslash\{u\})=$ Shade $F$.

We proceed exactly as in our above proof of Statement 1 up until the point where we define $F^{\prime}$. Insead of setting $F^{\prime}=F \cup\{u\}$, we now set $F^{\prime}=F \backslash\{u\}$. Combining $\alpha(F) \subseteq F$ with $u \notin \alpha(F)$, we obtain $\alpha(F) \subseteq F \backslash\{u\}=F^{\prime}$. Combining this with $F^{\prime}=F \backslash\{u\} \subseteq F \subseteq \tau(F)$, we see that $F^{\prime} \in$ $[\alpha(F), \tau(F)]$. From this, we can obtain Shade $\left(F^{\prime}\right)=$ Shade $F$ by the same argument that we used back in the proof of Statement 1. In view of $F^{\prime}=F \backslash\{u\}$, this rewrites as Shade $(F \backslash\{u\})=$ Shade $F$. This proves Statement 2.]

Now, we have proved Statements 1 and 2. Thus, the map Shade : $\mathcal{P}(E) \rightarrow \mathcal{P}(E)$ satisfies the two axioms in Definition 4.2. In other words, this map is a shade map. This proves Theorem 4.28 (a).

(b) Let $F \in \mathcal{P}(E)$. We must prove that $\alpha(F)=F \cap$ Shade $F$ and $\tau(F)=F \cup(E \backslash$ Shade $F)$.

As in the above proof of Statement 1, we can see that $\alpha(F) \subseteq F \subseteq \tau(F)$ and Shade $F=E \backslash$ $(\tau(F) \backslash \alpha(F))$ and $E \backslash$ Shade $F=\tau(F) \backslash \alpha(F)$. Hence, Lemma 4.29 (b) (applied to $X=\alpha(F)$ and $Y=F$ and $Z=\tau(F))$ yields that $F \cap(E \backslash(\tau(F) \backslash \alpha(F)))=\alpha(F)$. In view of Shade $F=$ $E \backslash(\tau(F) \backslash \alpha(F))$, this rewrites as $F \cap$ Shade $F=\alpha(F)$. In other words, $\alpha(F)=F \cap$ Shade $F$.

Furthermore, Lemma 4.29 (a) (applied to $X=\alpha(F)$ and $Y=F$ and $Z=\tau(F)$ ) yields that $F \cup$ $(\tau(F) \backslash \alpha(F))=\tau(F)$. In view of $E \backslash$ Shade $F=\tau(F) \backslash \alpha(F)$, this rewrites as $F \cup(E \backslash$ Shade $F)=$ $\tau(F)$. In other words, $\tau(F)=F \cup(E \backslash$ Shade $F)$. Thus, Theorem 4.28 (b) is proven.

(c) Each block of $\mathbf{P}$ has the form $[\alpha(F), \tau(F)]$ for some $F \in \mathcal{P}(E)$ (since it is a Boolean interval, thus nonempty, therefore contains some $F \in \mathcal{P}(E)$; but then it must be $[\alpha(F), \tau(F)]$ for this $F)$. Conversely, any set of the form $[\alpha(F), \tau(F)]$ is a block of $\mathbf{P}$ (by the definition of $[\alpha(F), \tau(F)]$ ). Combining these two facts, we conclude that the blocks of $\mathbf{P}$ are precisely the sets of the form $[\alpha(F), \tau(F)]$ with $F \in \mathcal{P}(E)$. But this is precisely the claim of Theorem 4.28 (c).

A converse to Theorem 4.28 is provided by the following theorem: 
Theorem 4.30. Let $E$ be a finite set. Let Shade $: \mathcal{P}(E) \rightarrow \mathcal{P}(E)$ be a shade map on $E$. Define a map $\alpha: \mathcal{P}(E) \rightarrow \mathcal{P}(E)$ by setting

$$
\alpha(F)=F \cap \text { Shade } F \quad \text { for any } F \in \mathcal{P}(E) .
$$

Define a map $\tau: \mathcal{P}(E) \rightarrow \mathcal{P}(E)$ by setting

$$
\tau(F)=F \cup(E \backslash \text { Shade } F) \quad \text { for any } F \in \mathcal{P}(E) .
$$

Let

$$
\mathbf{P}=\{[\alpha(F), \tau(F)] \mid F \in \mathcal{P}(E)\} .
$$

Then:

(a) We have $F \in[\alpha(F), \tau(F)]$ for any $F \in \mathcal{P}(E)$.

(b) If $F \in \mathcal{P}(E)$ and $G \in[\alpha(F), \tau(F)]$, then $[\alpha(F), \tau(F)]=[\alpha(G), \tau(G)]$.

(c) The set $\mathbf{P}$ is a Boolean interval partition of $\mathcal{P}(E)$.

(d) We have Shade $F=E \backslash(\tau(F) \backslash \alpha(F))$ for any $F \in \mathcal{P}(E)$.

To prove this theorem, we will need the following variant of Lemma 4.23 :

Lemma 4.31. Let $E$ be a set. Let Shade $: \mathcal{P}(E) \rightarrow \mathcal{P}(E)$ be a shade map. Let $A$ and $B$ be two subsets of $E$ such that $B$ is finite and $B \cap$ Shade $A=\varnothing$. Then, Shade $(A \backslash B)=$ Shade $A$.

Proof of Lemma 4.31: Induction on $|B|$, using Axiom 2 from Definition 2.6.

We will also use another simple set-theoretical lemma (easily checked using Venn diagrams):

Lemma 4.32. Let $E$ be a set. Let $X$ and $Y$ be two subsets of $E$. Then,

$$
E \backslash Y=(X \cup(E \backslash Y)) \backslash(X \cap Y) .
$$

Proof of Theorem 4.30: (a) Let $F \in \mathcal{P}(E)$. The definition of $\alpha$ yields $\alpha(F)=F \cap$ Shade $F \subseteq F$. The definition of $\tau$ yields $\tau(F)=F \cup(E \backslash$ Shade $F) \supseteq F$, so that $F \subseteq \tau(F)$. Thus, $\alpha(F) \subseteq F \subseteq \tau(F)$. In other words, $F \in[\alpha(F), \tau(F)]$. This proves Theorem 4.30 (a).

(b) Let $F \in \mathcal{P}(E)$ and $G \in[\alpha(F), \tau(F)]$. From $G \in[\alpha(F), \tau(F)]$, we obtain $\alpha(F) \subseteq G \subseteq$ $\tau(F)$. Thus, $G \subseteq \tau(F)=F \cup(E \backslash$ Shade $F)$ (by the definition of $\tau$ ). From this, straightforward set-theoretical reasoning leads to

$$
(G \backslash F) \cap \text { Shade } F=\varnothing .
$$

Furthermore, the definition of $\alpha$ yields $F \cap$ Shade $F=\alpha(F) \subseteq G$. From this, straightforward settheoretical reasoning leads to

$$
(F \backslash G) \cap \text { Shade } F=\varnothing .
$$

Hence, Lemma 4.31 (applied to $A=F$ and $B=F \backslash G$ ) yields Shade $(F \backslash(F \backslash G))=$ Shade $F$. In view of $F \backslash(F \backslash G)=F \cap G$, this rewrites as Shade $(F \cap G)=$ Shade $F$. Hence, (14) rewrites as

$$
(G \backslash F) \cap \text { Shade }(F \cap G)=\varnothing .
$$


Thus, Lemma 4.23 (applied to $A=F \cap G$ and $B=G \backslash F$ ) yields

Shade $((F \cap G) \cup(G \backslash F))=$ Shade $(F \cap G)=$ Shade $F$. In view of $(F \cap G) \cup(G \backslash F)=G$, this rewrites as

$$
\text { Shade } G=\text { Shade } F \text {. }
$$

By further set-theoretical reasoning, using the equalities (14) and (15) (and nothing else specific to the sets $F, G$ and Shade $F$ ), we can easily obtain

$$
F \cap \text { Shade } F=G \cap \text { Shade } F \quad \text { and } \quad F \cup(E \backslash \text { Shade } F)=G \cup(E \backslash \text { Shade } F) .
$$

In view of

$$
\begin{aligned}
& \alpha(F)=F \cap \text { Shade } F \quad \text { and } \quad \alpha(G)=G \cap \underbrace{\text { Shade } G}_{=\text {Shade } F}=G \cap \text { Shade } F \quad \text { and } \\
& \tau(F)=F \cup(E \backslash \text { Shade } F) \quad \text { and } \quad \tau(G)=G \cup(E \backslash \underbrace{\text { Shade } G}_{=\text {Shade } F})=G \cup(E \backslash \text { Shade } F),
\end{aligned}
$$

we can rewrite these two equalities as $\alpha(F)=\alpha(G)$ and $\tau(F)=\tau(G)$. Thus, $[\alpha(F), \tau(F)]=$ $[\alpha(G), \tau(G)]$. Theorem 4.30 (b) is thus proven.

(c) Clearly, $\mathbf{P}$ is a set of Boolean intervals of $\mathcal{P}(E)$. Moreover, the Boolean intervals $[\alpha(F), \tau(F)] \in$ $\mathbf{P}$ are pairwise disjoint (because if two of these intervals have some element $G$ in common, then Theorem 4.30 (b) yields that they must both equal $[\alpha(G), \beta(G)]$ ), and their union is $\mathcal{P}(E)$ (since every $F \in$ $\mathcal{P}(E)$ satisfies $F \in[\alpha(F), \tau(F)]$ by Theorem $4.30(\mathbf{a})$ ). Thus, $\mathbf{P}$ is a Boolean interval partition of $\mathcal{P}(E)$. This proves Theorem 4.30 (c).

(d) Let $F \in \mathcal{P}(E)$. Then, Lemma 4.32 (applied to $X=F$ and $Y=$ Shade $F$ ) yields

$$
E \backslash \text { Shade } F=\underbrace{(F \cup(E \backslash \text { Shade } F))}_{\begin{array}{c}
=\tau(F) \\
(\text { since } \tau(F)=F \cup(E \backslash \text { Shade } F))
\end{array}} \backslash \underbrace{(F \cap \text { Shade } F)}_{\begin{array}{c}
=\alpha(F) \\
(\text { since } \alpha(F)=F \cap \text { Shade } F)
\end{array}}=\tau(F) \backslash \alpha(F) .
$$

However, Shade $F$ is a subset of $E$; thus,

$$
\text { Shade } F=E \backslash \underbrace{(E \backslash \text { Shade } F)}_{=\tau(F) \backslash \alpha(F)}=E \backslash(\tau(F) \backslash \alpha(F)) .
$$

This proves Theorem 4.30 (d).

Combining Theorem 4.28 with Theorem 4.30, we obtain the following:

Theorem 4.33. Let $E$ be a finite set. Then, there is a bijection from the set

$$
\{\text { shade maps Shade }: \mathcal{P}(E) \rightarrow \mathcal{P}(E)\}
$$

to the set

$$
\{\text { Boolean interval partitions of } \mathcal{P}(E)\} \text {. }
$$

It sends each map Shade to the Boolean interval partition $\mathbf{P}$ defined in Theorem 4.30 (c). Its inverse map sends each Boolean interval partition $\mathbf{P}$ to the map Shade defined in Theorem 4.28. 
Proof of Theorem 4.33: This follows easily from Theorem 4.28 and Theorem 4.30.

Question 4.34. According to Theorem 4.18, any antimatroid gives rise to an inclusion-reversing shade map, which in turn gives rise to a Boolean interval partition by Theorem 4.30. Is this construction equivalent to the construction of a Boolean interval partition from an antimatroid described by Gordon and McMahon in Gordon and McMahon, 1997, Theorem 2.5)?

\section{The topological viewpoint}

Now we return to the setting of Section 11. We aim to reinterpret Theorem 2.11 in the terms of combinatorial topology (specifically, finite simplicial complexes) and strengthen it. We recall the definition of a simplicial complex: (iv)

Definition 5.1. Let $E$ be a finite set. A simplicial complex on ground set $E$ means a subset $\mathcal{A}$ of the power set of $E$ with the following property:

If $P \in \mathcal{A}$ and $Q \subseteq P$, then $Q \in \mathcal{A}$.

Thus, in terms of posets, a simplicial complex on ground set $E$ means a down-closed subset of the Boolean lattice on $E$. Note that a simplicial complex contains the empty set $\varnothing$ unless it is empty itself.

We refer to Kozlov 2020) for context and theory about simplicial complexes. We shall restrict ourselves to the few definitions relevant to what we will prove. The following is straightforward to check:

Proposition 5.2. Let us use the notations from Section 7 as well as Definition 2.6. Let $G$ be any subset of E. Let

$$
\mathcal{A}=\{F \subseteq E \mid G \nsubseteq \text { Shade } F\} .
$$

Then, $\mathcal{A}$ is a simplicial complex on ground set $E$.

Example 5.3. The following pictures illustrate this simplicial complex on an example.
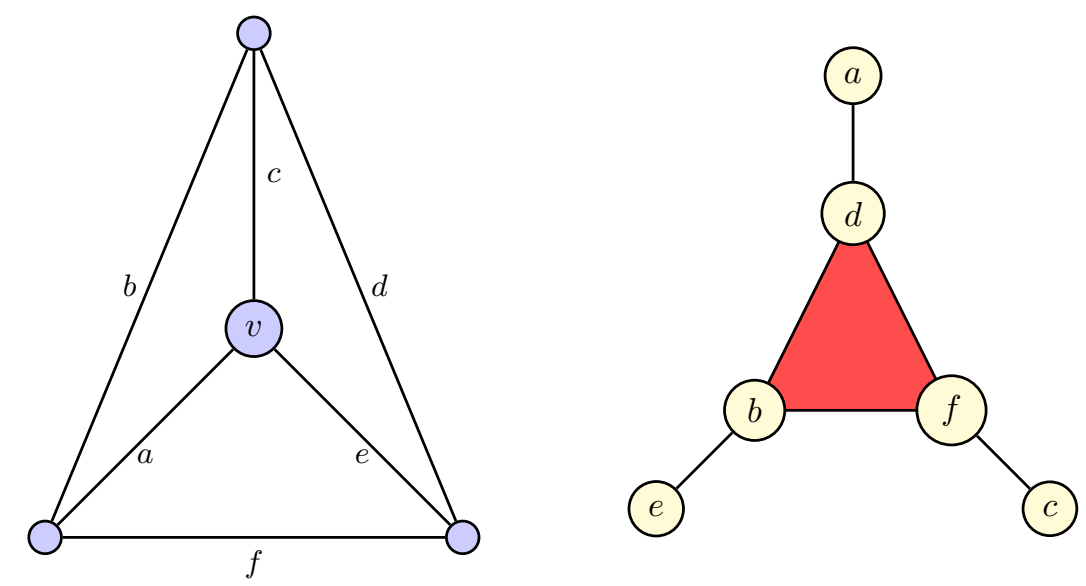

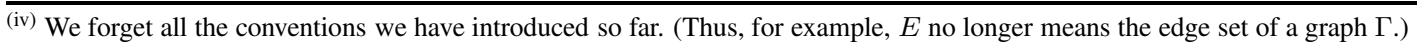


The left picture is a graph $\Gamma$ (with the vertex labelled $v$ playing the role of $v$ ), whereas the right picture shows the corresponding simplicial complex $\mathcal{A}$ for $G=E$ (that is, the simplicial complex whose faces are the subsets of $E$ that are not pandemic).

To state the main result of this section, we need the following definition $\mathrm{v}$ ):

Definition 5.4. Let $E$ be a finite set. Let $\mathcal{A}$ be a simplicial complex on ground set $E$.

(a) A complete matching $\mu$ of $\mathcal{A}$ is said to be acyclic if there exists no tuple $\left(B_{1}, B_{2}, \ldots, B_{n}\right)$ of distinct sets $B_{1}, B_{2}, \ldots, B_{n} \in \mathcal{A}$ with the property that $n \geq 2$ and that

$$
\mu\left(B_{i}\right) \prec B_{i} \quad \text { for each } i \in\{1,2, \ldots, n\}
$$

and

$$
\mu\left(B_{i}\right) \prec B_{i+1} \quad \text { for each } i \in\{1,2, \ldots, n-1\}
$$

and

$$
\mu\left(B_{n}\right) \prec B_{1} .
$$

(b) The simplicial complex $\mathcal{A}$ is said to be collapsible if it has an acyclic complete matching.

Note that our definition of an "acyclic complete matching" is a particular case of Kozlov, 2020, Definition 10.7). Our notion of "collapsible" is equivalent to the classical notion of "collapsible" (even though the latter is usually defined differently) because of (Kozlov, 2020, Theorem 10.9).

We now claim:

Theorem 5.5. Let us use the notations from Section 7 as well as Definition 2.6. Let $G$ be any subset of E. Define $\mathcal{A}$ as in (10). Then, the simplicial complex $\mathcal{A}$ is collapsible.

Collapsible simplicial complexes are well-behaved in various ways - in particular, they are contractible ((Kozlov, 2020, Corollary 9.19)), and thus have trivial homotopy and homology groups (in positive degrees). Moreover, the reduced Euler characteristic of any collapsible simplicial complex is 0 (for obvious reasons: having a complete matching suffices, even if it is not acyclic); thus, Theorem 2.11 follows from Theorem 5.5 .

Our proof of Theorem 5.5 will rely on the following simple lemma (whose proof is left as an exercise):

Lemma 5.6. Let $X$ and $Y$ be two sets, and let $u \in X \cap Y$. If $X \backslash\{u\} \prec Y$, then $X=Y$.

Proof of Theorem 5.5: We know from Proposition 5.2 that $\mathcal{A}$ is a simplicial complex. It remains to show that $\mathcal{A}$ is collapsible.

Note that our $\mathcal{A}$ is precisely the set $\mathcal{A}$ defined in the proof of Theorem 2.11 above.

We equip the finite set $E$ with a total order (chosen arbitrarily).

If $F \in \mathcal{A}$, then we define the edge $\varepsilon(F) \in G \backslash$ Shade $F$ as in the proof of Theorem 2.11.

We define a complete matching $\mu: \mathcal{A} \rightarrow \mathcal{A}$ of $\mathcal{A}$ as in the proof of Theorem 2.11. We shall now prove that this complete matching $\mu$ is acyclic.

Indeed, let $\left(B_{1}, B_{2}, \ldots, B_{n}\right)$ be a tuple of distinct sets $B_{1}, B_{2}, \ldots, B_{n} \in \mathcal{A}$ with the property that $n \geq 2$ and that

$$
\mu\left(B_{i}\right) \prec B_{i} \quad \text { for each } i \in\{1,2, \ldots, n\}
$$

(v) We continue using the definitions from Subsection 2.1, even though $E$ is now just an arbitrary finite set. 
and

$$
\mu\left(B_{i}\right) \prec B_{i+1} \quad \text { for each } i \in\{1,2, \ldots, n-1\}
$$

and

$$
\mu\left(B_{n}\right) \prec B_{1} .
$$

We shall derive a contradiction.

Set $B_{n+1}=B_{1}$. Then, combining (18) with (19), we conclude that

$$
\mu\left(B_{i}\right) \prec B_{i+1} \quad \text { for each } i \in\{1,2, \ldots, n\} .
$$

We now claim the following:

Claim 1: We have Shade $\left(B_{i}\right) \subseteq$ Shade $\left(B_{i+1}\right)$ for each $i \in\{1,2, \ldots, n\}$.

[Proof of Claim 1: Let $i \in\{1,2, \ldots, n\}$. From (17), we see that $\mu\left(B_{i}\right) \prec B_{i}$, so that $\varepsilon\left(B_{i}\right) \in B_{i}$ (by the definition of $\mu$ ). Set $u=\varepsilon\left(B_{i}\right)$. Then, the definition of $\mu$ yields $\mu\left(B_{i}\right)=B_{i} \backslash\{u\}$.

However, $u=\varepsilon\left(B_{i}\right) \in G \backslash$ Shade $\left(B_{i}\right)$ (by the definition of $\varepsilon\left(B_{i}\right)$ ). In other words, $u \in G$ and $u \notin$ Shade $\left(B_{i}\right)$. Therefore, (6) (applied to $\left.F=B_{i}\right)$ yields Shade $\left(B_{i} \backslash\{u\}\right)=$ Shade $\left(B_{i}\right)$. This can be rewritten as Shade $\left(\mu\left(B_{i}\right)\right)=$ Shade $\left(B_{i}\right)$ (since $\mu\left(B_{i}\right)=B_{i} \backslash\{u\}$ ).

But (20) yields $\mu\left(B_{i}\right) \prec B_{i+1}$, so that $\mu\left(B_{i}\right) \subseteq B_{i+1}$ and thus Shade $\left(\mu\left(B_{i}\right)\right) \subseteq$ Shade $\left(B_{i+1}\right)$ (by Lemma 2.8). In view of Shade $\left(\mu\left(B_{i}\right)\right)=\operatorname{Shade}\left(B_{i}\right)$, this can be rewritten as Shade $\left(B_{i}\right) \subseteq$ Shade $\left(B_{i+1}\right)$. This proves Claim 1.]

Claim 1 shows that Shade $\left(B_{1}\right) \subseteq$ Shade $\left(B_{2}\right) \subseteq \cdots \subseteq$ Shade $\left(B_{n}\right) \subseteq$ Shade $\left(B_{n+1}\right)$. This chain of inclusions is circular (since $\left.B_{n+1}=B_{1}\right)$; thus, it must be a chain of equalities. Hence, Shade $\left(B_{n}\right)=$ Shade $\left(B_{1}\right)$. Thus, $\varepsilon\left(B_{n}\right)=\varepsilon\left(B_{1}\right)$ (since $\varepsilon(F)$ depends only on Shade $F$, not on $F$ itself).

Set $u=\varepsilon\left(B_{n}\right)$. Thus, $u=\varepsilon\left(B_{n}\right)=\varepsilon\left(B_{1}\right)$.

We have $\mu\left(B_{n}\right) \prec B_{n}$ (by (17)); thus, the definition of $\mu$ yields $\varepsilon\left(B_{n}\right) \in B_{n}$ and $\mu\left(B_{n}\right)=B_{n} \backslash$ $\left\{\varepsilon\left(B_{n}\right)\right\}=B_{n} \backslash\{u\}\left(\right.$ since $\left.\varepsilon\left(B_{n}\right)=u\right)$.

However, $u=\varepsilon\left(B_{n}\right) \in B_{n}$ and similarly $u \in B_{1}$. Thus, $u \in B_{n} \cap B_{1}$. Also, $B_{n} \backslash\{u\}=\mu\left(B_{n}\right) \prec B_{1}$ (by (19)). Hence, Lemma 5.6 (applied to $X=B_{n}$ and $Y=B_{1}$ ) yields $B_{n}=B_{1}$. This contradicts the fact that the sets $B_{1}, B_{2}, \ldots, B_{n}$ are distinct (since $n \geq 2$ ).

Forget that we fixed $\left(B_{1}, B_{2}, \ldots, B_{n}\right)$. We thus have found a contradiction whenever $\left(B_{1}, B_{2}, \ldots, B_{n}\right)$ is a tuple of distinct sets $B_{1}, B_{2}, \ldots, B_{n} \in \mathcal{A}$ with the property that $n \geq 2$ and that $(17)$ and $(18)$ and (19). Hence, there exists no such tuple. In other words, the complete matching $\mu$ is acyclic. Therefore, the simplicial complex $\mathcal{A}$ is collapsible (by Definition 5.4 (b)). This finishes the proof of Theorem 5.5 .

The analogues of Proposition 5.2 and of Theorem 5.5 for vertex-infection (instead of usual infection) also hold (with the same proofs). More generally, Proposition 5.2 and Theorem 5.5 can be generalized to any inclusion-preserving shade map:

Theorem 5.7. Let $E$ be any set. Let Shade $: \mathcal{P}(E) \rightarrow \mathcal{P}(E)$ be an inclusion-preserving shade map on $E$. Let $G$ be any subset of $E$. Let

$$
\mathcal{A}=\{F \subseteq E \mid G \nsubseteq \text { Shade } F\} .
$$

Then:

(a) This $\mathcal{A}$ is a simplicial complex on ground set $E$.

(b) This simplicial complex $\mathcal{A}$ is collapsible. 
Proof: Part (a) is a straightforward generalization of Proposition 5.2, while part (b) is a straightforward generalization of Theorem 5.5. The proofs we gave above generalize (mutatis mutandis).

However, Theorem 5.7 cannot be lifted to the full generality of arbitrary shade maps, since $\mathcal{A}$ will generally not be a simplicial complex unless the shade map is inclusion-preserving. (However, for inclusionreversing shade maps, we can obtain a variant of Theorem 5.7 by applying Theorem 5.7 to the dual shade map Shade' from Proposition 4.7.)

\section{Open questions}

I shall now comment on two natural directions of research so far unexplored.

\subsection{The Alexander dual}

Any simplicial complex has an Alexander dual, which is defined as follows:

Definition 6.1. Let $E$ be a finite set. Let $\mathcal{A}$ be a simplicial complex on ground set $E$. Then, we define a new simplicial complex $\mathcal{A}^{\vee}$ on ground set $E$ by

$$
\mathcal{A}^{\vee}=\{F \subseteq E \mid E \backslash F \notin \mathcal{A}\} .
$$

(That is, $\mathcal{A}^{\vee}$ consists of those subsets of $E$ whose complements don't belong to $\mathcal{A}$.) This simplicial complex $\mathcal{A}^{\vee}$ is called the Alexander dual of $\mathcal{A}$.

It is well-known that a simplicial complex $\mathcal{A}$ and its Alexander dual $\mathcal{A}^{\vee}$ share many properties; in particular, the reduced homology of $\mathcal{A}$ is isomorphic to the reduced cohomology of $\mathcal{A}^{\vee}$ (see, e.g., (Björner and Tancer, 2009. Theorem 1.1)). However, the collapsibility and the homotopy types of $\mathcal{A}$ and $\mathcal{A}^{\vee}$ are not always related. Thus, the following question is suggested but not answered by Theorem 5.5:

Question 6.2. Let us use the notations from Section 7 as well as Definition 2.6 Let $G$ be any subset of E. Define $\mathcal{A}$ as in (16). Is the simplicial complex

$$
\mathcal{A}^{\vee}=\{F \subseteq E \mid G \subseteq \text { Shade }(E \backslash F)\}
$$

collapsible? Is it contractible?

\subsection{Several vertices $v$}

Elser's nuclei-based viewpoint in Elser 1984) (and (Dorpalen-Barry et al. 2021, Conjecture 9.1)) suggests yet another question.

Our definition of Shade $F$ (Definition 2.6), and the underlying notion of "infecting" an edge, implicitly relied on the choice of vertex $v$. It thus is advisable to rename the set Shade $F$ as $\operatorname{Shade}_{v} F$ and combine such sets for different values of $v$. In particular, we can define

$$
\mathcal{A}_{U}=\left\{F \subseteq E \mid G \nsubseteq \operatorname{Shade}_{v} F \text { for some } v \in U\right\}
$$

for any subset $U$ of $V$. This $\mathcal{A}_{U}$ is a simplicial complex (being the union of a family of simplicial complexes), and thus we can ask the same questions about it as we did about $\mathcal{A}$ :

Question 6.3. What can we say about the homotopy and discrete Morse theory of $\mathcal{A}_{U}$ ? What about its Alexander dual? 
For $G=E$ and $|U|>0$, this simplicial complex $\mathcal{A}_{U}$ is the Alexander dual of the " $U$-nucleus complex" $\Delta_{U}^{G}$ from (Dorpalen-Barry et al, 2021, Definition 3.2) (when $G$ is connected). If (Dorpalen-Barry et al., 2021, Conjecture 9.1 for $|U|>1$ ) is correct, then the homology of $\mathcal{A}_{U}$ with real coefficients should be concentrated in a single degree; this suggests the possible existence of an acyclic partial matching with all critical faces in one degree.

\section{Acknowledgements}

I thank Anders Björner, Galen Dorpalen-Barry, Dmitry Feichtner-Kozlov, Patricia Hersh, Sam Hopkins, Vic Reiner, Tom Roby and Richard Stanley for insightful conversations. A referee has greatly simplified the proof of Theorem 5.5. This research was supported through the programme "Oberwolfach Leibniz Fellows" by the Mathematisches Forschungsinstitut Oberwolfach in 2020. I am deeply grateful to the Institute for its hospitality during a none-too-hospitable time.

\section{References}

A. T. Benjamin and J. J. Quinn. An alternate approach to alternating sums: A method to DIE for. The College Mathematics Journal, 39(3):191-202, 2008. URL https: //scholarship.claremont.edu/cgi/viewcontent.cgi?referer=\&httpskedir= 1 \&article=1581\&context=hmc_fac_pub.

A. Björner and M. Tancer. Combinatorial Alexander duality - a short and elementary proof. Discrete Comput Geom, 42:586-593, 2009. doi: 10.1007/s00454-008-9102-x.

J. M. Brunat, A. Guedes de Oliveira, and M. Noy. Partitions of a finite boolean lattice into intervals. European Journal of Combinatorics, 30(8):1801-1809, Nov 2009. ISSN 0195-6698. doi: 10.1016/j. ejc.2008.12.010. URL http://dx.doi.org/10.1016/j.ejc.2008.12.010.

J. E. Dawson. A construction for a family of sets and its application to matroids. In L.L. MacAvaney (Ed.), Combinatorial Mathematics VIII (Geelong, 1980), volume 884 of Lect. Notes Math., pages 136-147. Springer, 1981.

K. Dedndreaj and P. Tittmann. On the activities and partitions of the vertex subsets of graphs, 2021. URL https://arxiv.org/abs/2008.02006v2.

G. Dorpalen-Barry, C. Hettle, D. C. Livingston, J. L. Martin, G. D. Nasr, J. Vega, and H. Whitlatch. A positivity phenomenon in Elser's gaussian-cluster percolation model. Journal of Combinatorial Theory, Series A, 179:105364, Apr 2021. ISSN 0097-3165. doi: 10.1016/j.jcta.2020.105364.

P. H. Edelman and R. E. Jamison. The theory of convex geometries. Geometriae Dedicata, 19:247-270, 1985. doi: 10.1007/BF00149365.

V. Elser. Gaussian-cluster models of percolation and self-avoiding walks. Journal of Physics A: Mathematical and General, 17(7):1515-1523, May 1984. ISSN 1361-6447. doi: 10.1088/0305-4470/17/7/ 019. URL http://dx.doi.org/10.1088/0305-4470/17/7/019

G. Gordon and E. McMahon. Interval partitions and activities for the greedoid tutte polynomial. Advances in Applied Mathematics, 18:33-49, 1997. 
D. Grinberg. The Elser nuclei sum revisited (arxiv version v8), 2021. URL http://arxiv.org/ abs/2009.11527v8.

B. Korte, L. Lovász, and R. Schrader. Greedoids. Algorithms and Combinatorics \#4. Springer, 1991. doi: 10.1007/978-3-642-58191-5.

D. N. Kozlov. Organized Collapse: An Introduction to Discrete Morse Theory, volume 207 of Graduate Studies in Mathematics. AMS, 2020. URL https://bookstore.ams.org/gsm-207.

B. Sagan. Combinatorics: The Art of Counting, volume 210 of Graduate Studies in Mathematics. AMS, 2020. URL https://users.math.msu.edu/users/bsagan/Books/Aoc/final.pdf. 